\title{
POPOLO, NAZIONE E PATRIA NELLE PRIME OPERE POLACCHE (1778-1794)
}

\author{
ALINA ŻÓRAWSKA-WITKOWSKA \\ Uniwersytet Warszawski
}

Izvleček: Konec 18. stoletja so pojmi ljudstvo, narod in domovina vplivali na prve poljske opere, $v$ katerih je bilo dogajanje postavljeno na podeželje. Libreti in glasba so bili prežeti z elementi govora in ljudskih pesmi različnih regij. Kralj Stanisław August je še posebej podpiral razsvetljensko idejo »ruralne opere«, povezano z izboljšanjem socialnega položaja kmetstva (ljudstva, naroda). Še več, idejo domovine lahko najdemo tudi $v$ delu z naslovom Kantata w dzień inauguracji statui króla Jana III (Varšava 1788).

Ključne besede: poljska ruralna opera, razsvetljenstvo, sarmatizem, Stanistaw August Poniatowski, Maciej Kamieński, Jan Stefani, Wojciech Bogusławski.
Abstract: At the end of the eighteenth century, the concepts of people, nation and homeland influenced the first Polish operas, whose topics were set in the countryside. Librettos and music incorporated authentic elements drawn from the speech and folk songs of different regions. In particular, King Stanisław August Poniatowski promoted the Enlightenment ideals of "rural opera", connected with the improvement of the wretched social conditions of the peasantry (i.e., the people or nation). Moreover, the concept of the fatherland is recognizable in Kantata w dzien inauguracji statui króla Jana III (Warsaw 1788).

Keywords: Polish rural opera, Enlightenment, Sarmatism, Stanisław August Poniatowski, Maciej Kamieński, Jan Stefani, Wojciech Bogusławski.

Il concetto di popolo e il canone musicale popolare assunsero un ruolo fondamentale nell'opera lirica polacca sin dai suoi inizi (1778-1794). Il primo concetto è palese soprattutto nei testi dei libretti, mentre il secondo emerge chiaramente dalle partiture conservatesi. In Polonia fu particolarmente popolare in quell'epoca un tipo di melodramma che gli studiosi definiscono "opera rurale". La trama dei lavori appartenenti a questo genere si svolge in un ambiente contadino, che permette di introdurre sia nel testo, sia nella musica e nella scenografia, un elemento di realismo, talvolta spiccato. Il carattere sincretico della tradizione popolare, ove convivono musica, danza e cerimonie, la rendeva particolarmente atta ad essere utilizzata nell'ambito dell'opera, genere di per sé sincretico.

La tradizione popolare in Polonia ha sempre avuto un ruolo di prim'ordine, rappresentando un'espressione dell'autoctonìa creata in gran parte dagli aristocratici. Questo fenomeno si accentuò nel diciottesimo secolo, trasformandosi in una particolare ideologia denominata sarmatismo (in sintesi, il culto dell'autoctono e dei valori nazionali), che si 
manifestò tramite una intensa "rusticizzazione" della cultura. Già nel secondo Settecento, però, per via della universalizzazione dei postulati dell'illuminismo, il concetto di popolo assunse nuovi significati. Agli illuminati, ossia agli intellettuali, artisti e magnati radunati attorno a Stanisław August Poniatowski che regnò dal 1764 al 1795, erano ovviamente note le posizioni di Jean-Jacques Rousseau e Johann Gottfried Herder, cosí come i Canti di Ossian (la prima traduzione polacca dalla versione francese, a cura di Ignacy Krasicki, apparve a Leopoli nel 1792-1793). L'attenzione nei confronti della tradizione popolare, non solo quella polacca ma anche degli altri popoli residenti sul territorio della Repubblica delle Due Nazioni (soprattutto al confine polacco-ruteno), era più antica e più profondamente radicata che non in Europa occidentale, e costituiva la summa di due orientamenti: quello "estetico", basato sulla conservazione delle tradizioni dell'antica Polonia, e quello storiografico, basato su alcuni elementi che furono considerati fonti per la storia della nazione. ${ }^{1}$ Lo stesso re sarebbe stato un appassionato cultore di musica popolare, come conferma uno dei cronisti di quell'epoca: "Egli vedeva le manifestazioni del popolo quali grandi opere degne dell'occhio regio. Perciò i signori, conoscendo quel gusto particolare, qualora fosse ospite a casa di uno di loro, mettevano in scena per lui delle nozze rustiche veritiere o immaginarie". ${ }^{2}$

\section{Popolo e nazione}

Per molto tempo, il termine nazione, nella Repubblica delle Due Nazioni, veniva riferito unicamente alla nobiltà. Lo stato comprendeva il Regno di Polonia (inclusa una parte dell'odierna Ucraina) e il Granducato di Lituania (composto dalle odierne Lituania e Bielorussia); era governato da un regime politico senza eguali, ossia una repubblica con un monarca eletto democraticamente. Il diritto di cittadinanza, tuttavia, interessava solo la nobiltà, un gruppo molto numeroso, composto dall' $8-10 \%$ della popolazione, e molto variegato per quanto riguarda la confessione (solo il 25\% era cattolico), inoltre lo status economico rivela che il 60\% dei signori non possedeva proprietà terriera. Quella nobiltà plurietnica (polacca, russa, lituana) era integrata grazie al mito della comune origine dal potente popolo degli antichi sarmati. Nella Repubblica delle Due Nazioni la borghesia era una minoranza debole, e i contadini costituivano la maggioranza del popolo soggetto, privo cioè di qualsiasi diritto pubblico essendo proprietà del signore. Un fatto confermato eloquentemente da atti di cessione dei musicisti, appartenenti alla classe infima, effettuati da magnati in favore di Antoni Tyzenhauz, tesoriore del Granducato di Lituania intorno al 1770. In uno di quegli atti leggiamo che veniva dato in dono non solo il musico, ma anche "la moglie sua e la progenie già esistente e quella futura".

Fu Stanisław August a tentare di riformare quelle drastiche ineguaglianze sociali. Il re considerava il teatro come uno dei mezzi più importanti della sua propaganda, utilizzandolo, come annunciò nel 1765, per combattere uno degli stereotipi polacchi, cioè

1 Górski, "Folklor", 154-160: 156.

2 Kitowicz, Pamiętniki, czyli Historia polska, 673.

3 Pl- Wagad, Archiwum Tyzenhauza, D-4/XVIII-7, f. 240. 
1'“oppressione delle genti di basso rango". ${ }^{4}$ Quando, vent'anni dopo, ereditò il corpo di ballo dal tesoriere Tyzenhauz, composto di giovani popolani, li liberò dalla sudditanza e concesse loro lo status di uomini liberi.

Il vasto stato polacco-lituano, plurietnico, plurilinguistico e pluriconfessionale, sotto il regno di Poniatowski tentò di "trasformarsi secondo il modello occidentale illuminato, che intendeva la nazione come comunità politica". Come ha ben definito lo storico Andrzej Walicki, il suo modello era lontano dall'“ideologia del risveglio etnico che ha caratterizzato i processi nazionalistici nei cosiddetti popoli senza storia, non possedenti uno stato, una lingua letteraria e una cultura superiore cristallizzata". ${ }^{5}$ Sulla base di tali azioni, la costituzione del 3 maggio 1791, la prima legge suprema in Europa e la seconda nel mondo dopo quella degli Stati Uniti d'America, ha introdotto il termine di nazione unitaria nel senso statale, comprendendo tutte le classi della società civile, quindi non solo l'aristocrazia, ma anche la borghesia e il ceto contadino (fatto, quest'ultimo, di straordinario rilievo). Uno degli articoli della costituzione fece persino uso del termine "nazione-popolo". ${ }^{6}$ In quell'occasione lo scrittore e pubblicista Franciszek Salezy Jezierski argomentava: "il popolo andrebbe nominato il primo stato della nazione, o parlando più chiaramente, la nazione assoluta". Lo stesso autore confermava che "l'aristocrazia in tutta Europa, in tutte le nazioni è simile [...]. Il popolo, invece, differenzia le nazioni, mantiene l'autoctonìa della lingua paterna, conserva le tradizioni ed è attaccato allo stesso modo di [concepire] la vita". Inoltre, aggiungeva che "nazione e governo della nazione sono cose distinte", portando ad esempio gli italiani: "La nazione italiana è una nazione molto distinta dalle altre, nonostante [...] non possieda governo, legge o potenza [...]; la nazione trasformata in regni, repubbliche, diverse forme di governo e regno, perse ciò che determina la serietà nazionale e che si manifesta nel carattere proprio del linguaggio, della musica, dell'architettura, della pittura". ${ }^{7}$

Secondo Jezierski, dunque, la nazione si articolava principalmente mediante la lingua e la cultura. Più o meno nello stesso tempo, una simile argomentazione fu presentata dall'eminente uomo politico e pubblicista Hugo Kołłątaj. Definendo il principio essenziale comune alla nazione, egli sosteneva: "la lingua paterna perfezionata, adoperata nell'istruzione e in tutti gli strumenti del governo, determina il carattere della nazione in modo molto più preciso che non l'abito; unisce in modo magistrale le diverse province". ${ }^{8}$ E il canonico del Duomo di Wawel Wacław Sierakowski, riferendosi alla questione che più ci interessa, chiedeva retoricamente: “Quando le altre grezze nazioni, poco propense alla melodia, danno cotanto valore alle loro lingue da scrivere con esse opere liriche, [perché] noi dovremmo scordare la nostra?".9

4 Listy Stanisława Augusta, 29.

5 Walicki, Idea narodu w polskiej myśli oświeceniowej, 10.

6 Augustyniak, Historia Polski 1572-1795, 316.

7 Salezy Jezierski, Niektóre wyrazy porzadkiem abecadła zebrane, 176-177.

8 Kołłątaj, Listy Anonima, vol. 1, 154.

9 Sierakowski, Sztuka muzyki, vol. I, 2. 


\section{L'opera polacca}

La nascita dell'opera polacca nell'ottavo decennio del Settecento fu una sorta di necessità storica. Si tratta di un fenomeno osservato anche in Austria (con il Singspiel), Danimarca, Svezia e Russia, emerso dai postulati dell'Illuminismo che esortavano a coltivare la lingua e l'arte nazionale in tutti i campi. In Polonia, l'opera fu anche un utile strumento per divulgare l'ideologia del potere presso la società. Il principe Michał Kazimierz Ogiński, librettista e compositore di opere in polacco rappresentate nella sua corte privata di Słonim (ora in Bielorussia) a partire dal 1771, nel 1779 scrisse in versi che l'opera:

Contiene in sé le verità fondamentali,

i principi del buon costume,

condanna il vizio, la virtù elogia,

scoraggia il cattivo costume ed esorta al buono. ${ }^{10}$

Il progetto di creare una forma d'arte propriamente nazionale sarebbe stato sostenuto dallo stesso re Stanisław August. Così almeno sostenne il compositore Maciej Kamieński, autore della prima opera del genere rappresentata in pubblico, Nędza uszczęśliwiona (La miseria resa felice, Varsavia 1778), su libretto di Franciszek Bohomolec e Wojciech Bogusławski, che ancora anni dopo si definiva con orgoglio l'autore della "primissima musica d'Opera Naz:[ionale] Orig:[inale]". ${ }^{11}$ Egli scrisse: "Siccome [...] gli italiani, i francesi e i tedeschi divertivano il pubblico con le loro opere, così volle il re che facessero anche i polacchi". ${ }^{12}$ Sul palcoscenico del teatro di Varsavia, infatti, si esibivano a turno le compagnie italiane, francesi, tedesche e polacche, e fu così che nacque La miseria resa felice, considerata dal coautore del libretto, Wojciech Bogusławski, un trionfo "sulla superstizione che il polacco non è portato all'opera lirica". ${ }^{13}$

Negli anni 1778-1794 fu creato un significativo repertorio di opere polacche. Nel solo teatro di Varsavia furono rappresentati circa sessanta titoli, di cui quaranta traduzioni di titoli stranieri (venticinque italiani, tredici francesi e due tedeschi), e diciotto lavori originali in polacco, creati cioè da scrittori e compositori locali. Aggiungendovi il repertorio di altri teatri della repubblica, il numero di opere originali polacche andrebbe stimato in almeno trentacinque lavori. Il principe Adam Kazimierz Czartoryski raccomandava che nel caso di opere tradotte da lingue straniere non si trattasse di traduzioni alla lettera, ma che vi fossero introdotti "costumi nostrani", ${ }^{14}$ e così avvenne. In tutte le opere di quel periodo, sia quelle originali sia quelle tradotte, le parti cantate, arie, "canzoni" e ensembles, sono intercalate da dialoghi in prosa, similmente al Singspiel tedesco e all'opéra comique francese.

\footnotetext{
${ }^{10}$ Prefazione al libretto dell'opera di Ogiński, Filozof zmieniony (Il filosofo cambiato), pagina non numerata.

${ }^{11}$ Gazeta Warszawska, 1814 no 49, 870.

${ }^{12}$ Kamieński, “Krótki rys [...] o egzystencji najpierwszej oryginalnej opery polskiej”, 411.

${ }^{13}$ Bogusławski, Dzieje Teatru Narodowego, 18.

${ }^{14}$ Czartoryski, prefazione al dramma Panna na wydaniu (Una signorina da maritare), 64.
} 


\section{La lingua dell'opera}

Al tempo di Stanisław August fu intrapreso un rinnovamento della lingua, deteriorata nel passato da maccheronismi barocchi. Nella vita pubblica l'aristocrazia usava il latino, mentre i magnati parlavano il francese, la lingua franca dell'epoca, che spesso conoscevano meglio della lingua materna. Fu concordato che il modello andava cercato sia negli scritti del poeta rinascimentale Jan Kochanowski, sia nell'idioma del popolo, liberato dai forestierismi. Per cui videro la luce i primi dizionari, grammaticali ed ortografici, e la lirica con il teatro si riferirono alla tradizione popolare. "Talvolta si andava a cercare la lingua popolare, la fonetica e il lessico dialettale, per caratterizzare la parlata specifica dei personaggi, sottolineare la loro origine sociale, la diversità degli ambienti; altre volte, si trattava di citare un testo popolare, riferirsi ai versi, allo stile, all'immaginario dei canti popolari nella lirica amorosa dell'epoca; altre volte ancora si trattava di introdurre la danza e la musica popolare, o certi elementi della tradizione cerimoniale del popolo ( $\mathrm{p}$. es. le nozze) e dei suoi costumi". ${ }^{15}$

Esempi di riferimento a modelli popolari si trovano nei libretti di Nędza uszczęśliwiona (La miseria resa felice), nella quale le protagoniste del popolo usano una parlata masoviana, Prostota cnotliwa (La semplicità virtuosa, Franciszek Bohomolec, Varsavia 1779?), dove tutti i personaggi, "sia nello stile, sia nel tono, devono parlare in modo popolare", Zośka, czyli Wiejskie zaloty (Sofia o Gli amori contadini, Stanisław Szymański, Varsavia 1780), nella quale i dialoghi parlati sono caratterizzati da una lingua grezza che imita piuttosto il gergo delle periferie di Varsavia che non quello popolare, mentre le parti cantate, pur essendo composte in lingua letteraria, nell'espressione sono chiaramente stilizzate sul modello della lirica popolare. E ancora in Agatka czyli Przyjazd pana (Agata o l'Arrivo del signore, Maciej Radziwiłł, Nieśwież 1784), ove i contadini dialogano in una lingua stilizzata sul modello popolare della Mazuria, mentre i nobili parlano la lingua letteraria, in Cud mniemany, czyli Krakowiacy i Górale (Il miracolo presunto, ossia i cracoviani ed i montanari, Wojciech Bogusławski, Varsavia 1794), opera che felicemente rappresenta la raffinatezza della tradizione popolare, quale fonte importante della cultura nazionale, sia dal punto di vista del materiale letterario sia musicale. ${ }^{16}$ Nel caso dei Krakowiacy $i$ Górale, i due gruppi etnici eponimi, tormentati da un palese conflitto, rappresentano il popolo polacco con la sua parlata e i suoi costumi. I dialetti adoperati dai cracoviani e dai montanari, tuttora molto diffusi, non costituiscono una lingua polacca concreta, ma rappresentano una sintesi "della lingua contadina con elementi caratteristici delle diverse regioni del paese". ${ }^{17}$

Si trattava indubbiamente di una strategia consapevole, consona alle azioni politiche della cerchia del re. Wojciech Bogusławski, autore del libretto di Krakowiacy i Górale, sottolineava la presenza di una tendenza nazionale nelle opere liriche polacche anteriori, ma confessava che ormai: "all'opera lirica polacca mancava quello che ad ogni nazione è più caro: [...] la nazionalità. Mi è occorsa l'idea di rappresentare in scena quei cracoviani

15 Górski, "Folklor", 158.

${ }^{16}$ Żórawska-Witkowska, "People, Nation and Fatherland in Three Polish Operas", 41-58.

${ }^{17}$ Raszewski, Bogusławski, vol. 1, 317. 
gioviali e rustici, che cantano coltivando i campi e cantano lottando per la patria. [...] Ho invocato la mia memoria e tutta la mia capacità di dipingere i loro costumi, espressioni, emozioni, parlate e divertimenti; [...] i cracoviani vinsero i cuori di tutti, accesero le menti di tutti. Le allusioni che tutti cercavano alla situazione attuale fecero sì che l'opera fosse proibita dopo tre rappresentazioni". ${ }^{18} \mathrm{Al}$ pubblico fu infatti evidente il contesto politico dei momenti caldi precedenti l'insurrezione antirussa del 1794. Si associavano quindi i cracoviani pacifici ai rappresentanti della nazione polacca, mentre i montanari, i quali aggredivano i cracoviani, ai russi che costituivano una minaccia per i polacchi.

Argomenti rustici, infine, anche se di stile più arcadico che realistico, erano presenti nelle opere rappresentate al teatro dei principi Izabela e Michał Kazimierz Czartoryski di Puławy (per esempio Zosinki e Marynki, del librettista Franciszek Karpiński con la musica di Wincenty Lessel).

\section{Realismo dei costumi e radicalismo sociale}

La trama delle opere citate si concentra sulle peripezie stereotipe di una coppia di amorosi, calate su uno sfondo realistico, o persino in un contesto di spiccato radicalismo sociale, che costituisce il loro tratto specifico. In Nędza uszczęśliwiona (1778) gli elementi di critica sociale sono ancora attenuati e l'ambiente contadino è rappresentato in modo convenzionale. L'unico elemento di critica sociale si osserva nella dichiarazione del borghese Jan, rivolta ad un contadino povero sebbene nobile di cuore:

\footnotetext{
Non conosci ancora i costumi dei Signori, che hanno sempre delle belle parole, belle storie che poco son vere.

Chi non conosce la miseria non sente pietà, beato chi si giova della loro grazia, beato chi di essa bisogno non ha. ${ }^{19}$
}

Nella Prostota cnotliwa (La semplicità virtuosa) l'ambiente contadino è dipinto in modo più crudo, e il libretto assume un tono da manifesto. La trama narra dell'esperimento effettuato da un nobile che ha liberato i suoi contadini e concesso loro dei terreni (nel territorio polacco annesso alla Russia, la liberazione dei contadini avvenne ufficialmente solo nel 1861). Secondo il nobile, uno dei due protagonisti dell'opera, "non è lecito che un uomo tenga un altro uguale a sé in servitù e lo tratti come bestiame". L'altro protagonista, il contadino Antek, intona un testo che la censura sicuramente non avrebbe autorizzato né a Parigi, né a Vienna:

Spietati assassini degli innocenti, non basta tenerci in servitú?

${ }^{18}$ Bogusławski, Dzieje Teatru Narodowego, 76-77.

${ }^{19}$ Id., Dzieła dramatyczne, vol. 12, 445. 
Ci ponete i vincoli anche sui cuori,

anche il bestiame vive meglio da voi. ${ }^{20}$

In Agatka (Maciej Radziwiłł, Nieśwież 1784) il proprietario della campagna apprezza il ruolo dei contadini, ammettendo che sono loro a consentire l'esistenza materiale dell'intera nazione: "Sono loro che ci nutrono, che ci danno il pane. [...] Del loro lavoro pagato con il sangue vivono tutti gli stati". ${ }^{21}$

\section{La musica}

Nell'epoca in esame, la varietà stilistica delle musiche delle diverse nazioni si attribuiva, seguendo il pensiero di Rousseau e Herder, alle caratteristiche timbriche, espressive e grammaticali delle diverse lingue. Ancora nel 1821 il compositore Karol Kurpiński scriveva: "Che cosa definisce la letteratura musicale nazionale? Il canto, perché si coniuga con la lingua paterna e vi assume un tratto caratteristico e nazionale. È diverso il timbro della lingua italiana da quella francese, tedesca o polacca; e chiunque approfondisca l'argomento sente che la differenza dei timbri si traduce nell'espressione del canto"’22: un'idea condivisa da Józef Elsner, il maestro di Fryderyk Chopin. Era diffusa, infatti, la convinzione che la musica su testo polacco di per sé era sufficiente a garantire la sua nazionalità, poiché si richiedeva ai compositori di adeguarsi all'intonazione della lingua, alle sue caratteristiche grammaticali, accentuative e timbriche.

L'introduzione di elementi nazionali nella musica dell'opera polacca del Settecento era legata alla necessità di rappresentare l'ambito contadino che, come già indicato, diventava spesso il luogo dell'azione drammatica. L'unico manoscritto originale, conservato della Nędza uszczęśliwiona, contiene una nota interessante del compositore Maciej Kamieński. Ai lettori Kamieński scrive: "Questi canti alla moda [enfasi originale, NdA] non sono composti per i critici, ma per l'uso dei polacchi". ${ }^{23}$ E infatti, come testimonia Bogusławski, alcuni brani dell'opera "venivano cantati ovunque e furono apprezzati ovunque". ${ }^{24}$ Anche se oggi vi è qualche difficoltà a individuare gli autentici elementi "rusticani", la musica fu qualificata con gli aggettivi "rustica e naturale", e Kamieński fu considerato il primo "che ebbe il coraggio in quel divertimento di indicare loro [i Polacchi] l'alba amena della nazionalità". 25

Kamieński fece un ulteriore passo avanti con Prostota uszczęśliwona (La semplicità virtuosa, la cui partitura non si è conservata), opera nella quale uno dei protagonisti di secondo piano doveva cantare, come indica una didascalia nel libretto, "un canto masoviano qualsiasi", ed anche "saltare", cioè ballare. Kamieński, inoltre, utilizzò numerosi

${ }^{20}$ Bohomolec, Prostota cnotliwa, pagina non numerata.

${ }^{21}$ Radziwiłł, Agatka, czyli Przyjazd pana, 497. Pl-Wn, manoscritto 6960.

${ }^{22}$ Kurpiński, “Odpowiedź panu G.”, 25.

${ }^{23}$ Kamieński, Nędza uszczęśliwiona, il manoscritto in Pl-Wtm.

${ }^{24}$ Bogusławski, Dzieje Teatru Narodowego, 20.

${ }^{25}$ Id., Dzieła dramatyczne, vol. 12, 422. 
elementi folklorici nella detta Zośka, czyli Wiejskie zaloty (Sofia, o gli amori contadini). Fino all'avvento dei Krakowiacy i Górale, Zośka godette di un successo enorme sulle scene polacche. Del resto è difficile immaginare che il libretto di quell'opera, così radicato nella lingua popolare (sia contadina che suburbana), non armonizzasse con la musica, di cui rimane solo una riduzione per pianoforte e le parti dell'orchestra, conservate a Leopoli (L'viv, Ucraina) nelle collezioni dell'antica Biblioteca Baworowski. Un riferimento alla pratica esecutiva dei canti popolari si osserva nella scelta del compositore di far precedere un preludio strumentale ad ogni pezzo vocale, di costruzione talmente semplice da non poterlo definire con il termine convenzionale di aria ma piuttosto di "canto". La provenienza popolare è anche plausibile nella musica che accompagna la canzone della vecchia Maryna Oj dadada, dada dada: una frase di quattro misure a ritmo di mazurka, ripetuta quattro volte. Si potrebbero citare molti altri esempi, basti la testimonianza di Bogusławski che sottolineava: "[Zośka] è scritta completamente nel gusto polacco" (equivalente a popolare). ${ }^{26}$ Un altro autore indicava che "quando si cantava la Zośka, la compagnia si rallegrava e si beveva un bicchiere di vino", ${ }^{27}$ anche se l'opera elogiava piuttosto la vodka e non il vino.

In Zośka, nell'arietta interpretata dalla protagonista eponima, Serce nie chłopiec (Il cuore non è un ragazzo), il verso relativo alla libertà ("La libertà gli è concessa una volta") si svolge su una melodia che nella struttura ritmica e intervallare si avvicina molto al secondo periodo della Mazurka di Dąbrowski, l'inno nazionale polacco composto nel 1797 a Reggio Emilia (va ricordato che la partitura di Zośka risale al 1780 circa). Un altro riferimento alle prime misure della Mazurka di Dabrowski si trova nella polacca dell'opera Żólta szlafmyca (La cuffia gialla, libretto di Franciszek Zabłocki, Varsavia 1783), composta dal musico reale Gaitano (Kajetan Meyer). Si potrebbe quindi arguire che l'inno nazionale si ispirò a un'altra melodia polacca rielaborata da Gaitano. Per Żótta szlafmyca il compositore utilizzò anche altri motivi strettamente popolari, tra cui il canto di Natale Lulajże Jеzипіи (Dormi Gesù Cristo), melodia genialmente reinterpretata anche da Chopin nello Scherzo in si minore op. 20.

Le manifestazioni più evidenti della nazionalità in musica furono le danze polacche: la polacca, la mazurka (con le sue varianti oberek e kujawiak), il krakowiak, le danze montanare. Nel ventesimo secolo, l'eminente critico Józef Sikorski riconobbe in quelle danze, "da tempo considerate nazionali", il "più alto grado di nazionalità". ${ }^{88}$ Particolarmente ricca di tali elementi si rivela la partitura di Cud mniemany, czyli Krakowiacy i Górale. ${ }^{29}$ I due gruppi in conflitto sono caratterizzati dall'uso di strumenti popolari in scena: le danze dei cracoviani sono accompagnate da un violinista e da uno zampognaro (I, 4), mentre i montanari dispongono di un complesso composto di flauti, tamburo, trombetta e scacciapensieri (I, 10). ${ }^{30}$ Oskar Kolberg, eminente etnografo polacco dell'Ottocento, esaminando i legami dell'opera con la musica popolare, trovò un numero di analogie dirette senza poter determinare se si trattasse di elementi ricavati dal folklore, oppure se

\footnotetext{
${ }^{26}$ Ibid., 20.

${ }^{27}$ Dąbrowski, "Niedole aktorów w XVIII wieku”, 245.

${ }^{28}$ Sikorski, "Halka", 3.

${ }^{29} \mathrm{In} \mathrm{Pl}-\mathrm{Wtm}$.

${ }^{30}$ Bogusławski, Cud albo Krakowiacy i Górale, 20, 46.
} 
nel comporre la musica Jan Stefani avesse imitato quella tradizione con tanto successo da essere successivamente adottata dal popolo. Kolberg citava tra l'altro l'esempio della polacca Rzadko to bywa w świecie (Succede di rado al mondo, I, 3), che riteneva "una versione [della polacca] stampata nella mia collezione di canti [...] e scritta così fluentemente che sarei propenso a considerare la versione di Stefani essere l'originale, e quella popolare, nonostante sia più semplice, esserne l'imitazione". ${ }^{31}$ Altri esempi di simili analogie si trovano nel krakowiak $O j d a d a d a$, oj da da da, tańcujmy wesoło (Oj da da da, oj da da da, balliamo lietamente) e nella mazurka Stała panna nad strumykiem (Una ragazza stava al ruscello), motivi molto vicini agli autentici canti del popolo.

Wincenty Lessel, maestro di cappella dei principi Izabela ed Adam Kazimierz Czartorysky, nel 1804 scrisse per il teatro di questi ultimi a Puławy una nuova musica per l'opera Dwaj strzelcy i mleczarka (Due cacciatori e la lattaia), tradotta in polacco dal francese e tuttavia assai diversa dall'originale (Les deux chasseurs et la laitière, Louis Anseaume, traduzione polacca di Jan Baudouin de Courtenay, 1779). Per l'occasione, Lessel comunicò a suo figlio Franciszek, allievo di Joseph Haydn, che avrebbe dovuto includere dei krakowiak e dei mazur. ${ }^{32}$

Elementi tipici dell'area di confine tra Polonia e Russia, quali la danza kozak e il canto epico-malinconico duma, sono stati utilizzati nel balletto del violinista del Teatro Nazionale Antoni Hart Kozaki (I cosacchi, Varsavia 1785), che godé di un'eccezionale popolarità. Infine, i riferimenti al già citato interesse di Stanisław August per le nozze popolari ricorrono nella commedia anonima Pan poznany (Il signore riconosciuto, Varsavia 1774), alla quale nel 1784 furono aggiunti "divertimenti e cerimonia di nozze, secondo il costume attuale dei contadini di Cracovia". ${ }^{33}$ Alla commedia fece seguito il balletto Krakowiacy i Kozaki (I cracoviani e i cosacchi), ${ }^{34}$ probabile adattamento dell'omonimo balletto.

\section{La patria}

La nozione di patria, meglio che in altri generi scenici, trovò la sua massima espressione artistica nella cantata drammatica, assai vicina all'opera lirica. Su ispirazione di Stanisław August Poniatowski, la Kantata na dzień inauguracji statui króla Jana III (Cantata per il giorno della inaugurazione della statua del re Giovanni III, libretto dello storico Adam Naruszewicz e musica di Maciej Kamieński) fu eseguita a Varsavia il 14 Settembre 1788. ${ }^{35}$ La forma ed il contenuto del libretto furono controllati dallo stesso re, poiché il lavoro doveva esibire elementi patriottici e storici nuovi per la Polonia. Sull'esempio del valoroso

${ }^{31}$ Kolberg, "Melodie ludowe w operze Jana Stefaniego", 361-363.

${ }^{32}$ Rudnicka Kruszewska, Wincenty Lessel. 112-114: "mit Musik von mir, [...] aber immer noch nach Krakowiaki, Mazuren riechen muß”.

${ }^{33}$ Bernacki, Teatr, dramat i muzyka za Stanisława Augusta, vol. 1, 309.

${ }^{34}$ Ibid.

${ }^{35}$ Libretto in: [Adam Naruszewicz], Opisanie festynu danego w Eazienkach [...], 16. Partitura in Pl-Wtm. 
Jan III Sobieski, l'eroe che sconfisse i turchi a Vienna nel 1683, i cittadini dovevano scoprire i loro sentimenti patriottici e ripetere le gesta degli antenati. Il re desiderava incitare l'intera nazione a partecipare alla guerra russo-turca e in cambio si aspettava di ricevere dalla zarina Caterina II alcune concessioni per attuare le riforme in Polonia. Per la prima volta nella storia, i quattro protagonisti della cantata, che portano i nomi di pastori greci, elogiano Sobieski in polacco con una serie di recitativi accompagnati, arie e ensembles. Il testo allude apertamente alla situazione del paese, e sottolinea la necessità di un rafforzamento militare dello stato, lamentando la discordia nazionale che avrebbe comportato il fallimento della patria. Kamieński fece uso di melodie molto note, per esempio una marcia dei tempi di Sobieski, e durante l'esecuzione queste furono ripetute dal pubblico.

La compresenza di contenuti politici e musicali nelle opere polacche del Settecento divenne una costante nel diciannovesimo secolo, quando la sudditanza dello stato innescò la lotta per il mantenimento della coscienza nazionale anche per il tramite delle arti. Agli stessi strumenti fece ancora ricorso Stanisław Moniuszko nelle opere Halka (libretto di Włodzimierz Wolski, Vilnius 1848, nuova versione Varsavia 1858) e Straszny dwór (Il castello dei fantasmi, libretto di Jan Chęciński, Varsavia 1865). Il patriottismo di stato, promosso da Poniatowski, ha avuto una evoluzione diversa da quello russo, fiorito a causa delle guerre napoleoniche. La continuità dell'opera nazionale in Polonia fu favorita dal lungo stato di eccezione, in cui venne a trovarsi il paese dopo la spartizione del suo territorio tra Prussia, Russia e Austria. Nata a corte per cementare l'unità della nazione sulla scorta delle tradizioni popolari, l'opera si tramutò in uno strumento di conservazione dell'identità dei polacchi, qualsiasi fosse il loro ceto sociale.

\section{Bibliografia}

AAugustyniak, Urszula. Historia Polski 1572-1795. Warszawa: Wydawnictwo Naukowe PWN, 2008.

Bernacki, Ludwik. Teatr, dramat i muzyka za Stanisława Augusta, vol. 1. Lwów:

Wydawnictwo Zakładu Narodowego imienia Ossolińskich, 1925.

Bogusławski, Wojciech. Dzieje Teatru Narodowego. Warszawa: Drukarnia N. Glücksberga, 1820.

Cud albo Krakowiacy i Górale, a cura di Mieczysław Klimowicz. Wrocław:

Zakład Narodowy imienia Ossolińskich, 2005.

Bohomolec, Franciszek. Prostota cnotliwa. Warszawa: Drukarnia Nadworna J. K. Mci, 1770.

Bohomolec, Franciszek e Bogusławski, Wojciech. Nędza uszczęśliwiona. In Wojciech

Bogusławski, Dzieła dramatyczne, vol. 12, 419-452. Warszawa: N. Glücksberg, 1823.

Czartoryski, Adam Kazimierz. Panna na wydaniu. Warszawa: Drukarnia J. K. Mci i

Rzeczpospolitej, 1771.

Dąbrowski, Stanisław. "Niedole aktorów w XVIII wieku”. In Pamiętnik Teatralny, 3-4

(1954): 245-254.

Gazeta Warszawska 49 (1814): 870.

Górski, Ryszard. "Folklor”. In Słownik literatury polskiego oświecenia, a cura di Teresa Kostkiewiczowa. 154-160. Wrocław: Zakład Narodowy imienia Ossolińskich, 1997. 
Jezierski, Franciszek Salezy. Niektóre wyrazy porządkiem abecadła zebrane. Warszawa: Michał Gröll, 1792.

Kamieński, Maciej. Nędza uszczęśliwiona, manoscritto in Pl-Wtm.

"Krótki rys [...] o egzystencji najpierwszej oryginalnej opery polskiej”.

In: Wejnert, Aleksander, "Stuletni jubileusz pierwszej opery polskiej”. In Biblioteka Warszawska, vol. 4 (1878): 410-422.

Kitowicz, Jędrzej. Pamiętniki, czyli Historia polska, a cura di Przemysława Matuszewska

e Zofia Lewinówna. Warszawa: Państwowy Instytut Wydawniczy, 1971.

Kolberg, Oskar. "Melodie ludowe w operze Jana Stefaniego: Krakowiacy i Górale”. Ruch Muzyczny 46 (1858): 361-363.

Kołłątaj, Hugo, Listy Anonima i Prawo polityczne narodu polskiego, vol. 1, a cura di

Bogusław Leśnodorski e Helena Wereszycka. Warszawa: Państwowe Wydawnictwo

Naukowe, 1954.

Kurpiński, Karol, “Odpowiedź panu G”. Tygodnik Muzyczny i Artystyczny, 7 (1821): 25-28. Listy Stanistawa Augusta do pani Geoffrin od roku 1764 do roku 1777, a cura di Lucjan

Siemieński. Kraków: Księgarnia J. M. Himmelblaua, 1876.

[Naruszewicz, Adam], Opisanie festynu danego w Eazienkach [...]. Warszawa: Piotr Dufour, 1788.

Ogiński, Michał Kazimierz. Filozof zmieniony. Wilno: s.e. 1779.

Pl- Wagad, Archiwum Tyzenhauza, D-4/XVIII-7, f. 240.

Radziwiłł, Maciej. Agatka, czyli Przyjazd pana. Pl-Wn, manoscritto 6960.

Raszewski, Zbigniew. Bogusławski, vol. 1. Warszawa: Państwowy Instytut Wydawniczy, 1972.

Rudnicka, Kruszewska Hanna. Wincenty Lessel. Szkic biograficzny na podstawie listów do syna. Kraków: Polskie Wydawnictwo Muzyczne, 1968.

Sierakowski, Wacław. Sztuka muzyki dla młodzieży krajowej, vol. I. Kraków: Drukarnia Szkoły Głównej Koronnej, 1795.

Sikorski, Józef. “Halka”. Ruch Muzyczny 1 (1858): 1-5.

Walicki, Andrzej. Idea narodu w polskiej myśli oświeceniowej. Warszawa: Polska Akademia Nauk Instytut Filozofii i Socjologii, 2000.

Żórawska-Witkowska, Alina. "People, Nation and Fatherland in Three Polish Operas: 'Cud mniemany, czyli Krakowiacy i Górale' (1794), 'Jadwiga, królowa polska' (1814), 'Król Łokietek, albo Wiśliczanki' (1818)'. In Nation and/or Homeland. Identity in $19^{\text {th }}$ Century Music and Literature between Central and Mediterranean Europe, a cura di Ivano Cavallini, 41-58. Milano: Mimesis, 2012.

"Lud, naród i ojczyzna w pierwszych operach polskich (1778-1794)”. In Operowy kontrapunkt. Libretto w Europie Środkowej i Wschodniej, a cura di Katarzyna Lisiecka e Barbara Judkowiak, 101-117. Poznań: Wydawnictwo Poznańskiego Towarzystwa Przyjaciół Nauk, 2014. 


\author{
PEOPLE, NATION AND HOMELAND \\ IN THE FIRST POLISH OPERAS (1778-1794)
}

\begin{abstract}
Summary
The concepts of people, nation and homeland influenced the librettos of the first Polish operas (1778-1794). The music had to be in agreement with the ideas expressed in the lyrics. However, the music has survived in only some cases. At that time, the opera style that scholars refer to as "rural opera" gained special popularity in Poland. Rural opera action, which was set in the countryside, provided a pretext for introducing a detailed presentation of reality in the literary, musical and staging layers. King Stanisław August Poniatowski, a patron of the Warsaw theatre, not only promoted the creation of the first Polish opera staged in public, Nędza uszczéśliwiona (Misery Made Happy; Franciszek Bohomolec/Wojciech Bogusławski - Maciej Kamieński, Warsaw 1778), but also supported Enlightenment ideals connected to improving peasants' social conditions. He also encouraged enlivening these operas with music stylised from rural examples, which plays a major role in the operas Zośka, czyli Wiejskie zaloty (Sophie, or Country Courtship; Stanisław Szymański - Maciej Kamieński, Warsaw 1779) and above all Cud mniemany, albo Krakowiacy $i$ Górale (The Presumed Miracle, or Cracovians and Highlanders; Wojciech Bogusławski - Jan Stefani, Warsaw 1794). The librettos of these operas and other similar ones astonish with their strong realism and social radicalism, which is enhanced in the musical layer by drawing upon folk sources. Moreover, Polish opera is affected by the idea of fatherland, as seen in the distinct example of Kantata $w$ dzień inauguracji statui króla Jana III (Cantata on the Day of the Unveiling of the Statue of King Jan III; Adam Naruszewicz - Maciej Kamieński, Warsaw 1788). It is clear that in the operatic compositions created during the reign of Stanisław August Poniatowski one can already find the ideals and musical means that would become the foundation of nineteenth-century Polish national opera (Stanisław Moniuszko).
\end{abstract}




\section{LJUDSTVO, NAROD IN DOMOVINA \\ V PRVIH POLJSKIH OPERAH (1778-1794)}

\section{Povzetek}

Pojmi ljudstvo, narod in domovina so vplivali na librete prvih poljskih oper (1778-1794). Glasba je morala biti v soglasju z idejami teh pojmov. Vendar pa se je glasba ohranila le v nekaterih primerih. V tistem času je opera, ki so ji raziskovalci pridali pridevnik »ruralna«, na Poljskem postala še posebno popularna. Dogajanje, postavljeno v podeželsko okolje, je omogočilo predstavitev tedanje realnosti na literarni, glasbeni in uprizoritveni ravni. Kralj Stanisław August Poniatowski, patron varšavskega gledališča, je spodbujal nastanek prve javno uprizorjene poljske opere Nędza uszczęśliwiona (Franciszek Bohomolec / Wojciech Bogusławski - Maciej Kamieński, Varšava 1778), pa tudi razsvetljenske ideje, povezane z izboljšanjem družbenega položaja ljudi, ki so bile ponazorjene s stilizirano ruralno glasbo in imajo pomembno vlogo v operah Zośka, czyli Wiejskie zaloty (Stanisław Szymański Maciej Kamieński, Varšava 1779) in predvsem Cud mniemany, albo Krakowiacy i Górale (Wojciech Bogusławski - Jan Stefani, Varšava 1794). Libreti omenjenih in tudi drugih oper nas presenečajo z močnim realizmom in socialnim radikalizmom, okrepljenim $\mathrm{V}$ glasbenih plasteh, ki črpajo iz ljudskih virov. Poleg tega je poljska opera vplivala na idejo domovine, katere izrazit primer je Kantata $w$ dzień inauguracji statui króla Jana III (Adam Naruszewicz - Maciej Kamieński, Varšava 1788). Ugotovimo lahko, da že med operami, nastalimi v času vlade Stanisława Augusta Poniatowskega, najdemo vzore in glasbena sredstva, ki so postali temelj poljske nacionalne opere 19. stoletja (Stanisław Moniuszko). 
